\title{
Nuevo sistema para la medida y control de la presión máxima de prensado en los alvéolos del molde: ISOPRESS ${ }^{\circledR}$
}

\author{
${ }^{1}$ A. POYATOS, ${ }^{1}$ R. BONAQUE, ${ }^{2}$ G. MALLOL, ${ }^{2}$ J. BOIX \\ ${ }^{1}$ MACER Cno. Estación s/n, 12550 Almazora \\ 2 Instituto de Tecnología Cerámica UJI (Castellón) Campus Universitario Riu Sec, Avda. de Vicent Sos Baynat s/n, 12006 Castellón
}

Este trabajo ha sido premiado con el Alfa de Plata en la Feria Internacional de Cerámica de Valencia CEVISAMA 2012

\begin{abstract}
La empresa MACER, en colaboración con el Instituto de Tecnología Cerámica, ha desarrollado el sistema ISOPRESS ${ }^{\circledR}$, un dispositivo de control integral que permite igualar de forma automática la presión máxima aplicada sobre el polvo contenido en cada uno de los alvéolos del molde. Este sistema está constituido por una serie de transductores de presión que se encuentran situados en los punzones isostáticos del propio molde. Con ellos es posible registrar en tiempo real la evolución de la presión media del aceite contenido en la cámara de compensación de cada punzón. Todos los transductores están conectados a un sistema de adquisición de datos que transfiere los valores de presión a un PC en el que se realiza el tratamiento de las señales para obtener el valor máximo de presión alcanzado durante el ciclo de prensado, en cada uno de los alvéolos. El sistema se completa con un software de control especialmente concebido que permite regular de forma individualizada la altura de la primera caída de cada uno de los punzones inferiores para garantizar la uniformidad de la presión aplicada en todos los alvéolos. ISOPRESS ${ }^{\circledR}$, al asegurar la constancia de la densidad aparente de todas las piezas procesadas, garantiza un único tamaño de pieza y minimiza los problemas de producción asociados a la variabilidad de la densidad aparente de los soportes.
\end{abstract}

Palabras clave: Densidad aparente, Prensado, Presión, Molde, Calibres

New system for measuring and controlling the maximum pressing pressure in the holes of the mould: ISOPRESS ${ }^{\circledR}$

The organization MACER, in collaboration with the Institute of Ceramic Technology, has developed the system ISOPRESS ${ }^{\circledR}$, an integrated control device that permits to equal automatically the maximum pressure applied on the powder contained in each of the holes of the mould. This system consists of a set of pressure transducers which are located in the isostatic punches of the mould itself. With them it is possible to register in real-time the evolution of the measured pressure of the oil contained in the compensation chamber of each punch. All the transducers are connected to a data acquisition system which transfers the pressure values to a PC which performs the signal processing to obtain the pressure maximum value reached during a pressing cycle, in each one of the holes. The system is completed with a control software especially developed, that permits to regulate individually the height of the first fall of each inferior punch to guarantee the uniformity of the pressure applied in all the holes. ISOPRESS ${ }^{\circledR}$, by assuring the constancy of the bulk density of all the pieces processed, guarantees a unique piece size and minimize production problems associated to the variability of the bulk density of the pieces.

Key words: Bulk density, pressing, pressure, mould, calibers

\section{INTRODUCCIÓN}

El procedimiento de control de la densidad aparente media empleado en la actualidad por la mayoría de los fabricantes de baldosas cerámicas persigue un doble objetivo. Por un lado, asegurar la constancia en el tiempo de la densidad aparente media de las piezas procesadas y, por otro lado, garantizar que todas las piezas obtenidas en un mismo ciclo de prensado posean una misma densidad aparente media. El primer objetivo se consigue mediante la modificación de la presión máxima del ciclo de prensado, en base a medidas de la humedad del polvo atomizado y de la densidad aparente en húmedo de los soportes. El segundo objetivo se alcanza tradicionalmente gracias a la incorporación de finas láminas metálicas de diferentes espesores entre los punzones y la placa portapunzones. Actuando de este modo se altera la distribución de las presiones aplicadas en los diferentes huecos del molde y, por tanto, es posible controlar la densidad aparente media alcanzada por cada soporte al finalizar el ciclo de prensado.

El control de la densidad aparente mediante la regulación de la presión máxima de prensado, en base a las variaciones de humedad del polvo atomizado, es un procedimiento que hoy en día puede ser completamente automatizado, tal y como lo hace MACER a través de su sistema de control CALIBREUNICO $^{\circledR}$. En cambio, el control de la densidad 
aparente media de las piezas obtenidas en un mismo ciclo de prensado se realiza todavía de una forma manual y discontinua. Por esta razón, esta forma de operar no permite actualmente asegurar la constancia de la densidad aparente media de todos los soportes obtenidos en cada prensada.

En los últimos años, han aparecido en el mercado varios dispositivos que intentan corregir las diferencias de densidad aparente entre las piezas conformadas en un mismo ciclo de prensado. Por un lado, existen los llamados moldes de placa isostática en los cuales los bloquetos no se apoyan directamente sobre la bancada del molde, sino que reposan sobre una cámara de aceite común mediante la cual se compensan las diferencias de presión que pueden aparecer entre los diferentes alvéolos. Este tipo de moldes, por el momento, no han tenido una gran aceptación debido a su gran complejidad mecánica y al hecho de que la regulación de presiones se realiza a costa de generar diferencias en el espesor medio de las piezas.

Por otro lado, también se han empezado a comercializar los conocidos como moldes de bloquetos móviles. Estos moldes presentan la peculiaridad de poder modificar de forma individualizada la posición de la primera caída de cada bloqueto, lo cual permite cambiar la cantidad de polvo alimentada en cada alvéolo, y con ello, la presión efectiva realmente aplicada en el mismo. Este sistema presenta la ventaja de no crear diferencias de espesor entre las piezas y tener una complejidad mecánica menor que la de los moldes de placa isostática. Sin embargo, hasta el momento, su control es manual y requieren la actuación de un operario que, en base a medidas rutinarias de la densidad aparente de los soportes, regule las posiciones de consigna de los bloquetos para igualar las densidades aparentes.

La densidad aparente media en seco de los soportes prensados depende, para una misma composición del polvo atomizado y una distribución de tamaños de partícula dentro de los márgenes habituales de trabajo, de la presión máxima aplicada sobre el polvo y de su humedad. La relación entre estas tres variables constituye el conocido como diagrama de compactación de la composición.

Aunque el diagrama de compactación es ampliamente utilizado en el control de la operación de prensado, éste no es suficiente para comprender el funcionamiento de los sistemas de regulación de la carga por bloquetos móviles, ya que no permite identificar el efecto de otras variables que, junto a la humedad y la presión, intervienen en la operación de prensado, como por ejemplo, la densidad del lecho de polvo tras el llenado del alveolo, el espesor inicial del mismo o el espesor final del soporte. Así pues, puede demostrarse que la presión aplicada por la prensa sobre el polvo se distribuye en los diferentes huecos en función del espesor inicial del lecho y de la densidad inicial del mismo, siendo el espesor inicial en cada uno de los huecos la variable que permite regular la presión aplicada en cada uno de ellos y, con ello, la densidad aparente de los soportes secos.
Por ello, la empresa MACER, con el fin de dar una solución a las diferencias de densidad aparente media generadas entre las piezas obtenidas en un mismo ciclo de prensado, ha desarrollado el sistema de control ISOPRESS ${ }^{\circledR}$. Este dispositivo mide la presión de prensado en cada uno de los alvéolos del molde y emplea dicha presión como variable de control de un sistema de regulación, basado en la modificación individualizada de la cantidad de polvo atomizado alimentado a cada hueco, que mantiene constante la presión máxima aplicada en todos los alvéolos y, por consiguiente, la densidad aparente media de todas las piezas procesadas.

El carácter continuo y totalmente automatizado del sistema ISOPRESS ${ }^{\circledR}$ lo convierten en una herramienta de gran utilidad y sencilla utilización para abordar problemas relacionados con una incorrecta densidad aparente en seco de los soportes prensados. La utilización del sistema implica un importante ahorro de costes de producción y limita enormemente los tiempos muertos de operación al reducir, e incluso eliminar, los ajustes mediante métodos tradicionales, que es necesario realizar en el molde para asegurar la constancia de las densidades aparentes.

La utilización de ISOPRESS ${ }^{\circledR}$ es totalmente compatible con la aplicación CALIBREUNICO ${ }^{\circledR}$ presentada por MACER en la anterior edición de CEVISAMA 2010 y galardonada con el premio Alfa de Oro. La conjunción de ambos sistemas permite regular de forma automática, y al mismo tiempo, la presión máxima aplicada por la prensa con el fin de compensar las posibles fluctuaciones de la humedad del polvo atomizado y la presión máxima realmente aplicada sobre el polvo contenido en cada alvéolo, mediante la regulación individualizada de la primera caída de los punzones.

Tanto el sistema ISOPRESS $^{\circledR}$, como el sistema CALIBREUNICO ${ }^{\circledR}$, son unos dispositivos industriales de asistencia en la etapa de conformado fruto de un importante trabajo de I+D. Estos sistemas proporcionan a los productos comercializados por MACER un carácter innovador y distintivo no aportado hasta el momento por el equipamiento complementario del prensado existente a día de hoy en el mercado. Ambos desarrollos son claro ejemplo de la fuerte apuesta realizada por MACER en los últimos años para dotar a sus productos de un carácter distintivo que aporte importante ventajas y mejoras a sus clientes con respecto a la oferta tradicionalmente existente.

\section{DESCRIPCIÓN DEL SISTEMA DE CONTROL}

El sistema ISOPRESS ${ }^{\circledR}$ está constituido por una serie de punzones isostáticos (tantos como piezas se prensan en un mismo ciclo de prensado (salidas)) que se encuentran instrumentados mediante unos transductores de presión capaces de medir la presión en el interior del lecho de aceite, que constituye la compensación hidráulica del propio

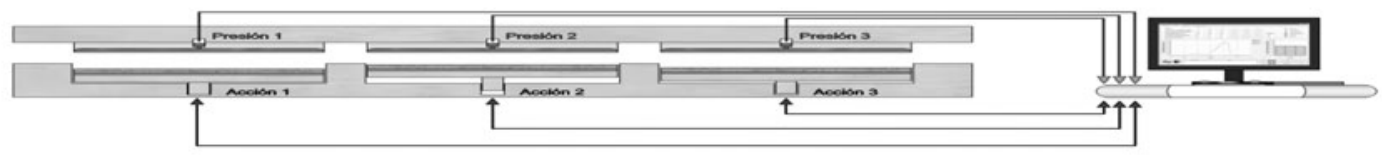

Figura 1. Esquema de la aplicación ISOPRESS ${ }^{\circledR}$ instalada a bordo de un molde penetrante de tres salidas. 
punzón. Como viene siendo habitual, en esta aplicación los punzones isostáticos son aquellos que se encargan de conformar la cara no vista de los soportes. De este modo, en los moldes penetrantes los punzones instrumentados son los que se encuentran en la parte superior del molde, mientras que en los conocidos como "moldes dobles", los punzones instrumentados se sitúan en la parte inferior.

Como puede observarse en el esquema de la Figura 1, las señales generadas por cada uno de los transductores de presión son transmitidas a una tarjeta de adquisición de datos de alta velocidad que se encarga de muestrear a elevada frecuencia la presión ejercida sobre el polvo contenido en cada alvéolo, a lo largo de cada ciclo de prensado.

Los datos registrados por la tarjeta de adquisición de datos son transferidos a un software de control especialmente concebido que aplica una serie de algoritmos matemáticos para el tratamiento de la señal recogida. Este procesamiento de datos permite obtener el valor máximo de la presión aplicada en cada alvéolo y en cada ciclo de prensado.

En la Figura 2 se muestra una captura de la pantalla principal del software de ISOPRESS ${ }^{\circledR}$ trabajando en una prensa equipada con un molde de 4 salidas de tamaño 350 $\mathrm{mm} \times 350 \mathrm{~mm}$. En dicha interfaz de usuario el operario puede ver en todo momento el ciclo de prensado experimentado por el polvo contenido en cada uno de los alvéolos y un gráfico de barras mostrando el valor de la presión máxima alcanzada en cada hueco en el último ciclo. Trabajando sobre un molde con bloquetos móviles, la aplicación proporciona información sobre la posición efectiva de cada uno de los bloquetos. Además, si sobre la prensa se encuentra trabajando un sistema de CALIBREUNICO ${ }^{\circledR}$, la medida de la humedad de las piezas proporcionada por este último es empleada para calcular, a partir del diagrama de compactación de la composición, la densidad aparente media obtenida en cada uno de los soportes recién prensados.

Desde esta misma pantalla, el operario de la prensa puede seleccionar de una manera sencilla el estado de funcionamiento del dispositivo (manual o automático) y acceder a otras pantallas de la aplicación, como por ejemplo, la que muestra la evolución de las presiones máximas registradas en cada alvéolo a lo largo del tiempo (ver Figura 3).

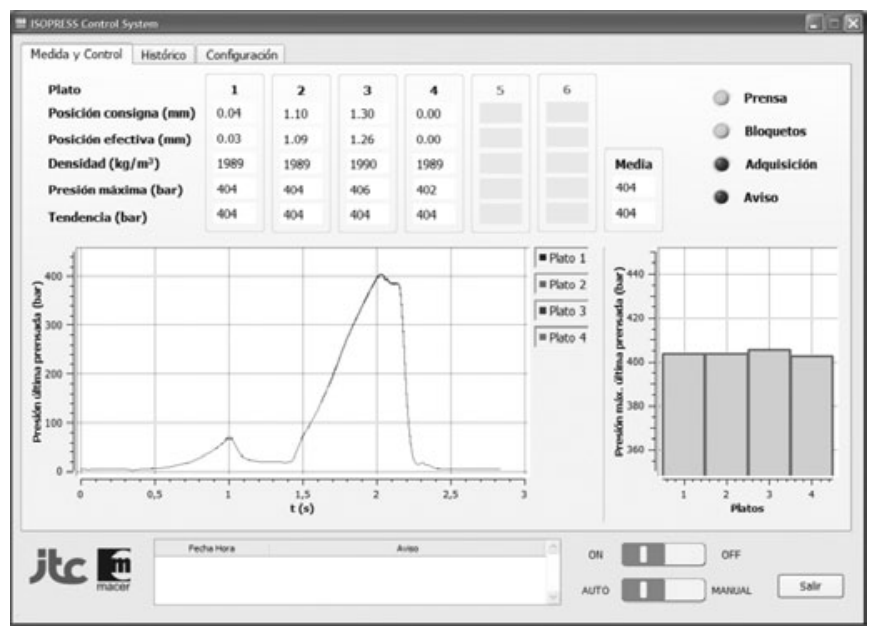

Figura 2. Pantalla principal de la aplicación ISOPRESS ${ }^{\circledR}$.

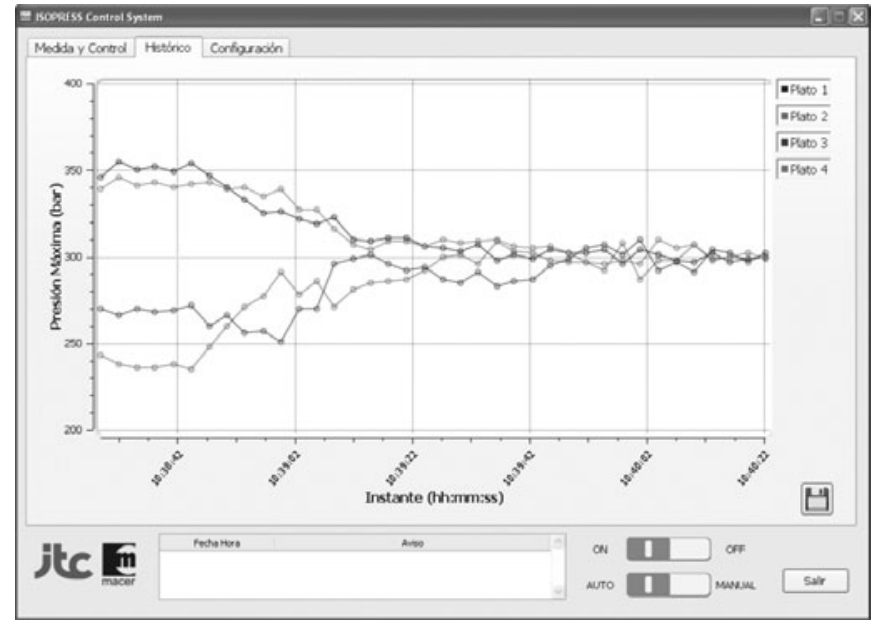

Figura 3. Evolución histórica de las presiones máximas registradas en cada alvéolo.

El funcionamiento del sistema se completa estableciendo comunicación con el molde de bloquetos móviles para modificar de forma individualizada la posición de la primera caída de cada uno de los bloquetos, con el fin de mantener la presión máxima de prensado de cada alvéolo dentro de un rango de variación preestablecido. Los parámetros característicos de la acción de control que se encarga de regular la carga de cada alvéolo para garantizar la igualdad de presiones pueden ser modificados desde la pantalla de configuración.

Cuando el sistema de control está actuando sobre la carga de polvo en cada alvéolo y las presiones se encuentran dentro del rango de variación permitido, el gráfico de barras de la pantalla principal aparece en color verde. En cambio, si el sistema funciona en modo manual y alguno de los alvéolos recibe una presión que se sitúa fuera de los límites definidos, dicho gráfico aparece en color rojo, tal y como se observa en la Figura 4, donde existe una diferencia de más de 50 bar entre la presión registrada en el punzón 1 y la medida en el punzón 2. En este mismo gráfico puede observarse como,

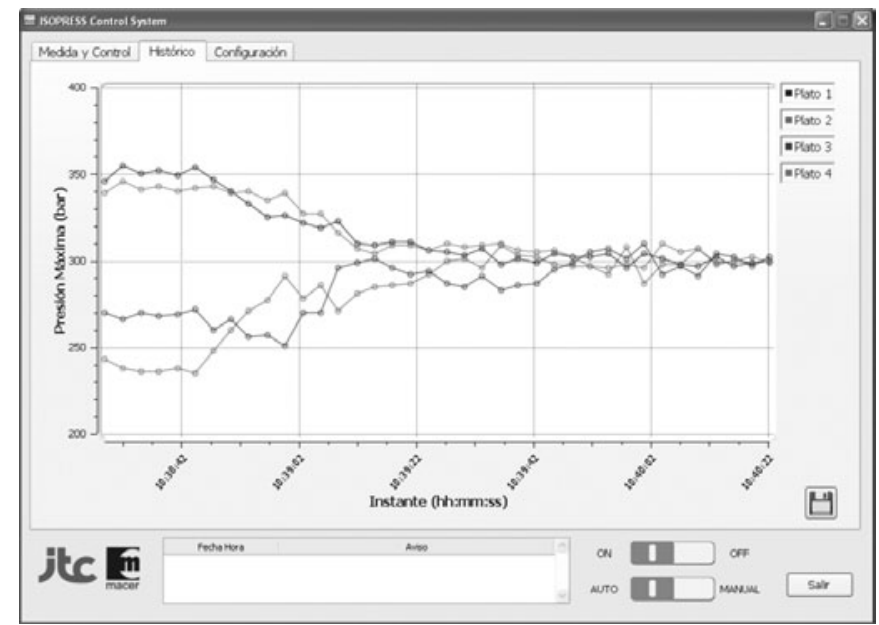

Figura 4. Pantalla principal de la aplicación ISOPRESS ${ }^{\circledR}$ mostrando una prensada con una mala distribución de la presión entre alvéolos. 


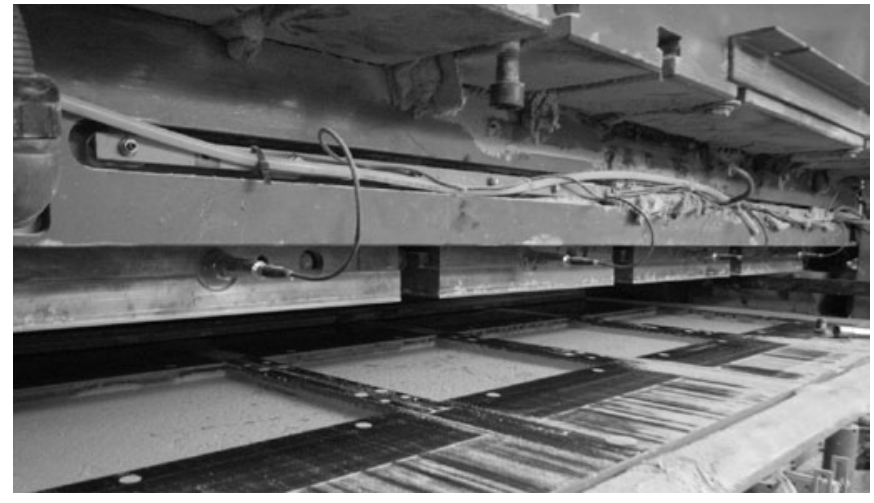

Figura 5. Punzones isostáticos dotados de transductores de presión en un molde de 4 salidas de $330 \mathrm{~mm} \times 330 \mathrm{~mm}$ de formato nominal equipado con el sistema ISOPRESS ${ }^{\circledast}$.

trabajando en modo automático, la acción de control efectuada en cada prensada se muestra, en forma de una flecha verde, en el indicativo del plato. En el ejemplo visualizado, los platos 1,3 y 4 estarían aumentando su posición de consigna para hacer disminuir su presión con respecto a la del plato 2, que se encuentra muy por debajo del valor medio de presión aplicada y, por lo tanto, está generando piezas con una densidad aparente inferior.

\section{VIABILIDAD INDUSTRIAL DE LA INNOVACIÓN PRESENTADA}

El sistema ISOPRESS ${ }^{\circledR}$ ha sido probado con éxito en un molde penetrante de cuatro salidas empleado en la fabricación de baldosas cerámicas de gres porcelánico de tamaño nominal $330 \mathrm{~mm}$ x $330 \mathrm{~mm}$. En la imagen de la Figura 5 pueden apreciarse los cuatro sensores de presión ubicados en los punzones superiores del molde, así como el cableado empleado para transmitir las señales hasta el PC de control.

Tras determinar las condiciones óptimas de muestreo mediante una serie de pruebas preliminares, se realizaron maniobras para validar el sistema de medida desarrollado. Estas maniobras consistieron en adquirir, durante varios ciclos de prensado, los valores de presión en los alvéolos del molde para compararlos con los valores de presión proporcionados por el transductor de presión instalado en el circuito hidráulico de la prensa.

En la Figura 6 se representa la variación de la presión en los alvéolos del molde y en el circuito hidráulico de la prensa durante dos ciclos de prensado completos. Durante el ciclo correspondiente al gráfico de la izquierda, el sistema de corrección de la posición de la primera caída de los bloquetos móviles permaneció desactivado, dando como resultado una distribución de presiones muy heterogénea, con una diferencia de presiones máximas entre alvéolos de 78 bar. Durante el ciclo mostrado a la derecha se activó la corrección por bloquetos móviles, lo cual proporcionó una distribución de presiones mucho más homogénea con tan sólo 8 bar de diferencia máxima entre alvéolos. Es interesante destacar que, aunque la actuación del sistema de corrección de la primera caída modifica la distribución de presiones en los alvéolos, el valor medio de estas presiones apenas se modifica (434 bar en el primer ciclo frente a 435 bar en el segundo), ya que la presión máxima aplicada en el circuito hidráulico de la prensa es la misma en los dos casos.

En el gráfico de la Figura 7 se muestra la evolución de las presiones máximas correspondientes a los cuatro huecos del molde a lo largo de 15 minutos de producción. Durante este periodo se recogieron las piezas prensadas en tres condiciones de funcionamiento diferentes, referenciadas en el gráfico como A, B y C, con el fin de determinar su densidad aparente y su tamaño tras la cocción.

La condición A hace referencia a una situación en la que la prensa estaba trabajando con el sistema ISOPRESS ${ }^{\circledast}$ desactivado, pero con el molde de bloquetos móviles configurado de tal forma que la presión aplicada sobre el alveolo 2 era ligeramente inferior a la de los otros tres alvéolos. En la condición B el sistema ISOPRESS ${ }^{\circledR}$ se encontraba trabajando de forma automática con el fin de homogenizar la distribución de presiones y en la condición C, trabajando también en modo automático, se aumentó la presión de prensado (ver Figura 7 (der.) para producir un cambio en la densidad aparente de los soportes.

Como puede apreciarse, al iniciarse el periodo de experimentación, la presión media registrada en los alvéolos de la prensa disminuyó progresivamente, como consecuencia de la regulación de la presión hidráulica llevada a cabo por el sistema de control automático CALIBREUNICO ${ }^{\circledR}$. Dicha acción de control fue motivada por el aumento de la humedad
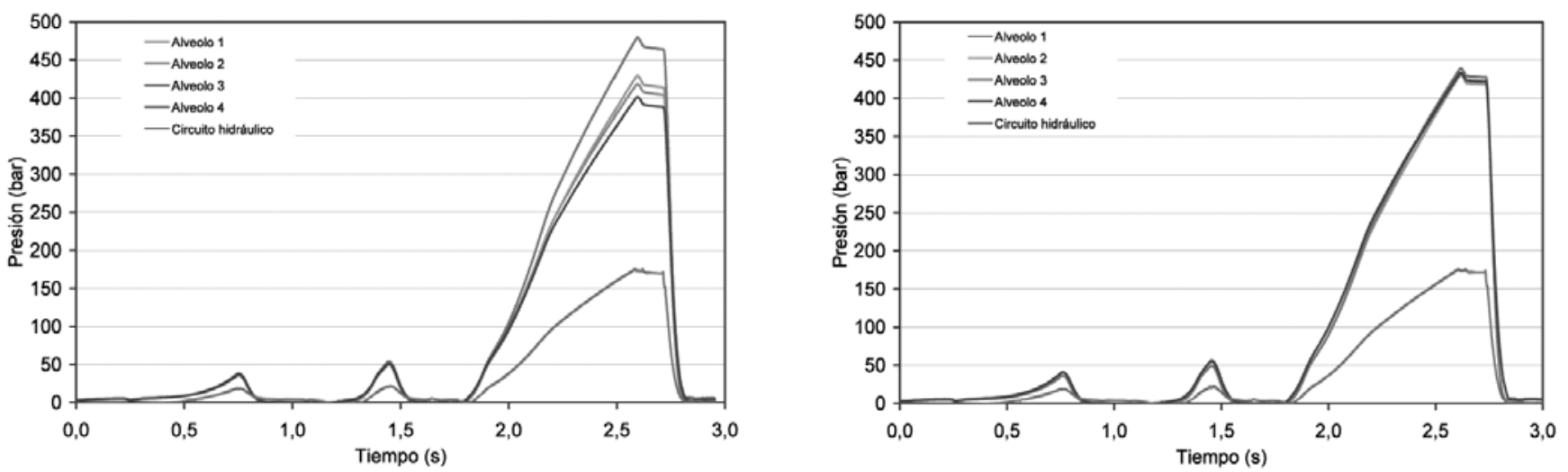

Figura 6. Evolución de las presiones registradas durante un ciclo de prensado sin corrección de la carga (izq.) y un ciclo de prensado con corrección (der.). 
TABla 1. PRESIÓN MÁXIMA, DENSIDAD APARENTE EN HÚMEDO Y TAMAÑO COCIDO DE LAS PIEZAS PROCESADAS EN DIFERENTES CONDICIONES DE OPERACIÓN.

\begin{tabular}{|c|c|c|c|c|c|c|c|c|c|c|c|c|}
\hline \multirow{2}{*}{$\begin{array}{l}\text { Condición } \\
\text { Alveolo } \\
\end{array}$} & \multicolumn{4}{|c|}{ A } & \multicolumn{4}{|c|}{ B } & \multicolumn{4}{|c|}{ C } \\
\hline & 1 & 2 & 3 & 4 & 1 & 2 & 3 & 4 & 1 & 2 & 3 & 4 \\
\hline Posición bloqueto (mm) & 0,07 & 0,00 & 0,05 & 0,04 & 0,13 & 0,00 & 0,08 & 0,07 & 0,13 & 0,00 & 0,08 & 0,07 \\
\hline$P_{1}$ (bar) & 439 & 425 & 437 & 436 & 429 & 432 & 429 & 430 & 470 & 470 & 471 & 471 \\
\hline $\mathrm{D}_{2}\left(\mathrm{~kg} / \mathrm{m}^{3}\right)$ & 2090 & 2090 & 2088 & 2088 & 2099 & 2099 & 2099 & 2099 & 2116 & 2117 & 2117 & 2116 \\
\hline $\mathrm{D}_{3}\left(\mathrm{~kg} / \mathrm{m}^{3}\right)$ & 1940 & 1940 & 1938 & 1938 & 1941 & 1941 & 1941 & 1941 & 1957 & 1958 & 1958 & 1957 \\
\hline $\mathrm{L}_{4}(\mathrm{~mm})$ & 335,1 & 334,3 & 334,9 & 335,0 & 335,3 & 335,1 & 335,1 & 335,3 & 335,5 & 335,5 & 335,6 & 335,6 \\
\hline Calibre & 4 & 3 & 4 & 4 & 4 & 4 & 4 & 4 & 5 & 5 & 5 & 5 \\
\hline$\Delta \mathbf{P}_{1 \max }($ bar $)$ & \multicolumn{4}{|c|}{14} & \multicolumn{4}{|c|}{3} & \multicolumn{4}{|c|}{1} \\
\hline$\Delta \mathrm{D}_{3 \max }\left(\mathrm{kg} / \mathrm{m}^{3}\right)$ & \multicolumn{4}{|c|}{2} & \multicolumn{4}{|c|}{0} & \multicolumn{4}{|c|}{1} \\
\hline$\Delta \mathbf{L}_{4 \max }(\mathrm{mm})$ & \multicolumn{4}{|c|}{0,8} & \multicolumn{4}{|c|}{0,2} & \multicolumn{4}{|c|}{0,1} \\
\hline
\end{tabular}

del polvo atomizado observado entre la 13:05 h y la 13:10 h (ver Figura 7 (der.)).

En la Tabla 1 se recogen la presión máxima, la densidad aparente en húmedo y el tamaño de las piezas tras la cocción, para las tres condiciones de operación evaluadas. Junto con estas variables también se muestra la posición de la primera caída de cada bloqueto móvil, tomando como posición de referencia la del momento de aplicación de la presión, la densidad aparente en seco, calculada teniendo en cuenta la humedad del polvo en el momento de recoger las piezas, y el calibre de las mismas.

En la condición A, debido a la menor presión aplicada sobre el polvo contenido en el alveolo 2, el tamaño de esta pieza tras la cocción era inferior al del resto de las piezas, para las cuales la presión máxima registrada fue prácticamente la misma. Como resultado de este comportamiento, las piezas procesadas en el alveolo 2 eran de un calibre inferior (calibre 3) al de las otras tres (calibre 4). Es interesante destacar que, si bien las diferencias de presión entre alvéolos eran evidentes, las diferencias de tamaño observadas no hubiesen podido ser detectadas a partir de las propias medidas de densidad aparente en húmedo realizadas en planta. En efecto, para una variación de la presión máxima aplicada de hasta 14 bares, que conducía a una diferencia de tamaños de $0,8 \mathrm{~mm}$, prácticamente la tolerancia de un calibre, que para este formato se suele fijar en $1 \mathrm{~mm}$, las diferencias de densidad aparente en húmedo medidas no superaban los $2 \mathrm{~kg} / \mathrm{m}^{3}$. Este hecho, pone de manifiesto que la medida de las presiones máximas registradas en los alvéolos del molde puede llegar a ser incluso más precisas que la propia medida de densidad aparente por inmersión en mercurio. De hecho, si bien el método de inmersión en mercurio posee una precisión máxima de $\pm 4 \mathrm{~kg} / \mathrm{m}^{3}$, los sensores de presión instalados permiten determinar la presión registrada sobre el polvo con una precisión de \pm 2 bar, lo cual, según el diagrama de compactación, para un polvo y humedad determinados equivaldría aproximadamente a $\pm 1 \mathrm{~kg} / \mathrm{m}^{3}$.

En la situación B, al trabajar en modo automático el sistema de control ISOPRESS ${ }^{\circledR}$ se disminuyó el recorrido de la primera caída de los bloquetos 1, 3 y 4, lo cual permitió disminuir el espesor inicial del lecho de polvo en estos huecos con respecto al espesor inicial del polvo en el alveolo 2. Fruto de esta maniobra se homogeneizó la distribución de presiones en todos los alvéolos, situándose la desviación máxima de
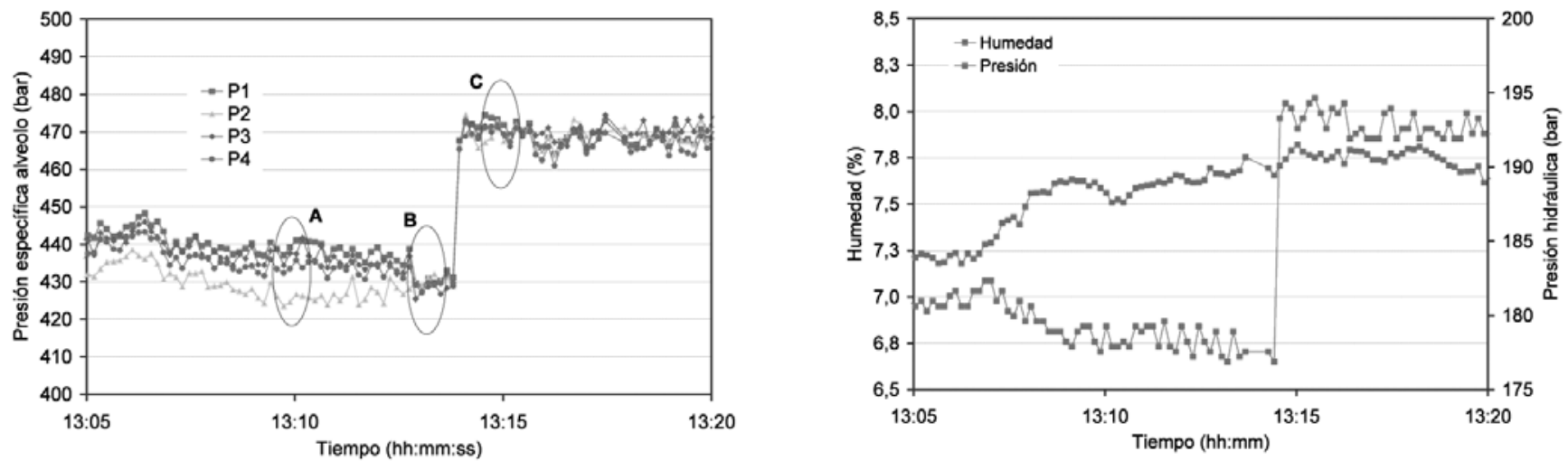

Figura 7. Evolución en el tiempo de las presiones específicas máximas registradas (izq.) y de la humedad del polvo atomizado y la presión en el circuito hidráulico (der.) durante 15 minutos. 
presión entre huecos en tal sólo 3 bar. Como resultado de esta acción, el tamaño de las piezas procesadas en el alveolo 2 aumentó significativamente y todas las piezas prensadas pudieron clasificarse en el calibre 4.

Finalmente, en la situación C, al aumentar la presión de prensado, modificando la consigna de densidad en el sistema de CALIBREUNICO $^{\circledR}$ de la prensa, la homogeneidad en la distribución de presiones alcanzada en la situación B se siguió manteniendo. Ahora bien, debido al aumento de la densidad aparente de los soportes procesados, el tamaño de las piezas tras la cocción fue ligeramente superior, situándose todas ellas en el calibre 5 .

\section{CONCLUSIONES}

A modo de conclusiones en los esquemas de la Figura 8 se resumen las ventajas más importantes que aporta la incorporación del sistema ISOPRESS ${ }^{\circledR}$ en un molde para la fabricación de baldosas cerámicas:

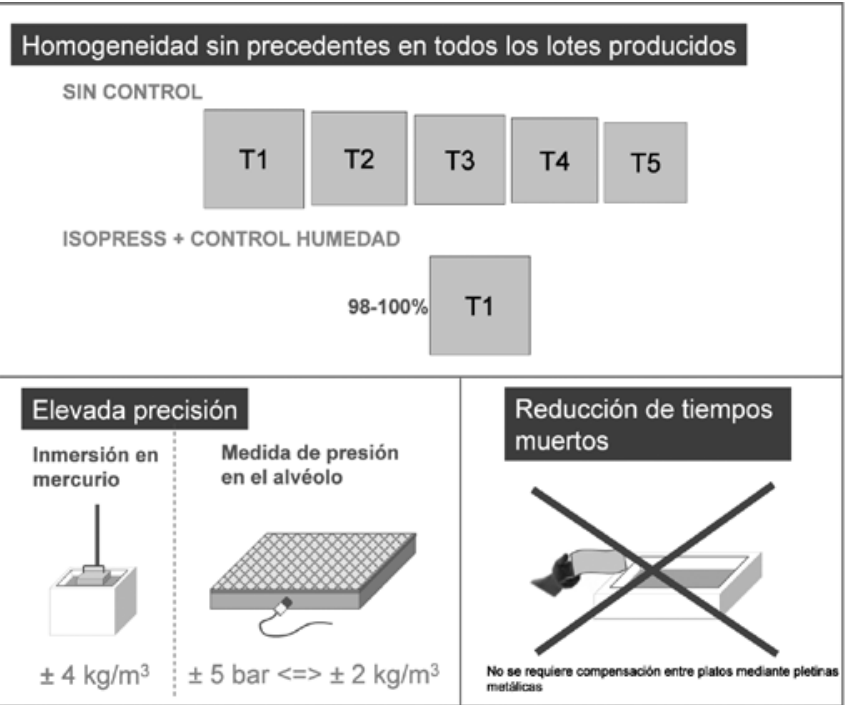

Figura 8. Ventajas aportadas por el sistema ISOPRESS ${ }^{\circledast}$
- Trabajando conjuntamente con el sistema CALIBREUNI$\mathrm{CO}^{\circledR}$, la constancia asegurada en la densidad aparente media de todos los soportes recién prensados garantiza una gran homogeneidad en la distribución de tamaños de las baldosas, al mismo tiempo que minimiza enormemente los problemas asociados a la variabilidad de la densidad aparente de los soportes.

- La medida de la presión de prensado en los alvéolos de la prensa para identificar posibles variaciones de la densidad aparente de los soportes ha demostrado ser incluso más precisa que la propia medida de la densidad aparente con alguno de los métodos de medida de la densidad aparente convencionales.

- La utilización del sistema ISOPRESS ${ }^{\circledR}$ disminuye considerablemente los tiempos muertos de operación al permitir alcanzar de forma rápida y automática una situación de estabilidad, en cuanto a la distribución de densidades aparentes entre platos se refiere. ISOPRESS ${ }^{\circledast}$ garantiza una correcta distribución de la presión de prensado en todos los alvéolos del molde sin necesidad de recurrir a métodos más costosos e imprecisos, como por ejemplo, la tradicional incorporación de pletinas metálicas entre el punzón y la placa portapunzones.

\section{AGRADECIMIENTOS}

Los autores de este trabajo agradecen al Instituto de la Pequeña y Mediana Industria Valenciana (IMPIVA) la financiación aportada para su realización, a través del programa de Investigación y Desarrollo Tecnológico.

\section{BIBLIOGRAFÍA}

(1) AMORÓS, J.L. Pastas cerámicas para pavimentos de monococción: Influencia de las variables de prensado sobre las propiedades de la pieza en crudo y sobre su comportamiento durante el prensado y la cocción. Valencia: Universidad. 1987, p.61 [Tesis doctoral]

(2) AMORÓS, J.L. et al. La operación de prensado en la fabricación de pavimento por monococción: I Influencia de la naturaleza del polvo de prensas sobre las propiedades de la pieza en crudo. Bol. Soc. Esp. Ceram. Vidrio. 27(5), 273-282, 1988

(3) AMORÓS, J.L. et al. Variables en la compactación de soportes cerámicos de pavimento y revestimiento. Técnica Cerámica, 105, 792-812, 1982

(4) MALLOL, G. Control y automatización en la industria cerámica: evolución y perspectivas. En: Qualicer 2006: IX Congreso Mundial de la Calidad del Azulejo y del Pavimento Cerámico. Castellón: Cámara Oficial de Comercio, Industria y Navegación, 2006. pp. Con47-Con72.

(5) POYATOS, A. et al. Nuevo sistema y metodología para la eliminación de los calibres en el proceso de fabricación de baldosas cerámicas. Bol. Soc. Esp . Ceram. Vidrio. 49(2), 147-151, 2010

(6) AMORÓS, J.L. et al. Mejora de la estabilidad dimensional de piezas de gres porcelánico a través de la medida en continuo de la humedad de los soportes prensados. Cerámica Información, 311, 117-126, 2004.

(7) AMORÓS, J.L. et al. Técnicas experimentales del control de la compactación de pavimentos y revestimientos cerámicos. Cerám. cristal, 94, 27-32, 1985. 